\title{
Three dimensional vectorial imaging of surface phonon polaritons
}

Xiaoyan $\mathrm{Li}^{1}$, Georg Haberfehlner ${ }^{2}$, Ulrich Hohenester ${ }^{3}$, Odile Stéphan ${ }^{4}$, Gerald Kothleitner ${ }^{5}$ and Mathieu Kociak $^{6}$

${ }^{1}$ CNRS, Orsay, Ile-de-France, France, ${ }^{2}$ Graz University, United States, ${ }^{3}$ Department of Physics, University of Graz, Graz, Austria, United States, ${ }^{4}$ Laboratoire de physique des solides CNRS, United States, ${ }^{5}$ Institute of Electron Microscopy and Nanoanalysis, Graz University of Technology, United States, ${ }^{6} \mathrm{CNRS}$, Orsay, Ile-de-France, United States

Surface phonon polaritons (SPhPs) are mixed electromagnetic and optical phonons waves that propagate at the surface of ionic materials (1). They strongly influence the optical and thermal behavior of nanomaterials. For example, they are responsible for highly coherent emission of $\mathrm{SiC}$ upon heating, in stark contrast with the conventional incoherent black-body radiation (2). They also induce enhanced thermal conduction in thin membranes (3) or heat transfer between two nanosurfaces (4). These applications rely on the nanostructuration of the electromagnetic field in the vicinity of surfaces of metamaterials or nanoparticles. Designing or even engineering the electro-magnetic local density of states (EMLDOS) for specific functionalities require therefore the unambiguous visualization of such field modulations at the nanometer scale. Recently, EELS in a scanning transmission electron microscope (STEM) made it possible to measure phonons spectra at the nanometer $(5,6)$, then atomic scales (7). Nevertheless, they were restricted to 2D imaging, and not able to reveal the complete three-dimensional vectorial picture of their electromagnetic density of states. Using a highly monochromated electron beam in a scanning transmission electron microscope, we could visualize varying $\mathrm{SPhPs}$ signatures from nanoscale $\mathrm{MgO}$ cubes as a function of the beam position, energy-loss and tilt angle, see Figure 1. Following early works on plasmons $(8,9)$, the SPhPs response was described in terms of eigenmodes and used to tomographically reconstruct the phononic surface electromagnetic fields of the object (10). Such 3D information promises novel insights in nanoscale physical phenomena and is invaluable to the design and optimization of nanostructures for fascinating new uses.

\section{Acknowledgements}

This work has received support from the National Agency for Research under the program of future investment TEMPOS-CHROMATEM with the Reference No. ANR-10-EQPX-50. This project has received funding from the european union's horizon 2020 research and innovation programme under grant agreement no 823717 (ESTEEM3) and 101017720 (EBEAM), and from the Austrian Science fund FWF under project P 31264. 
(a)

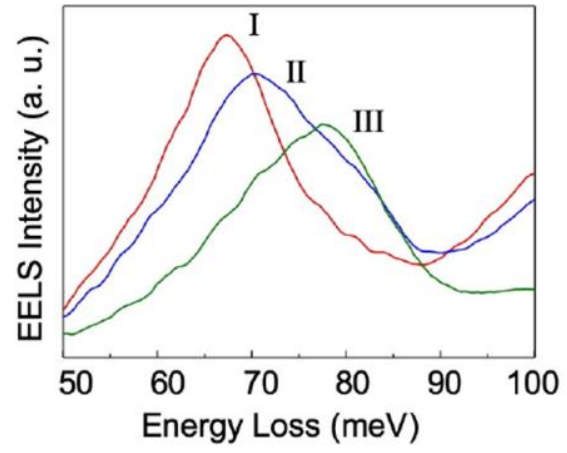

(b)
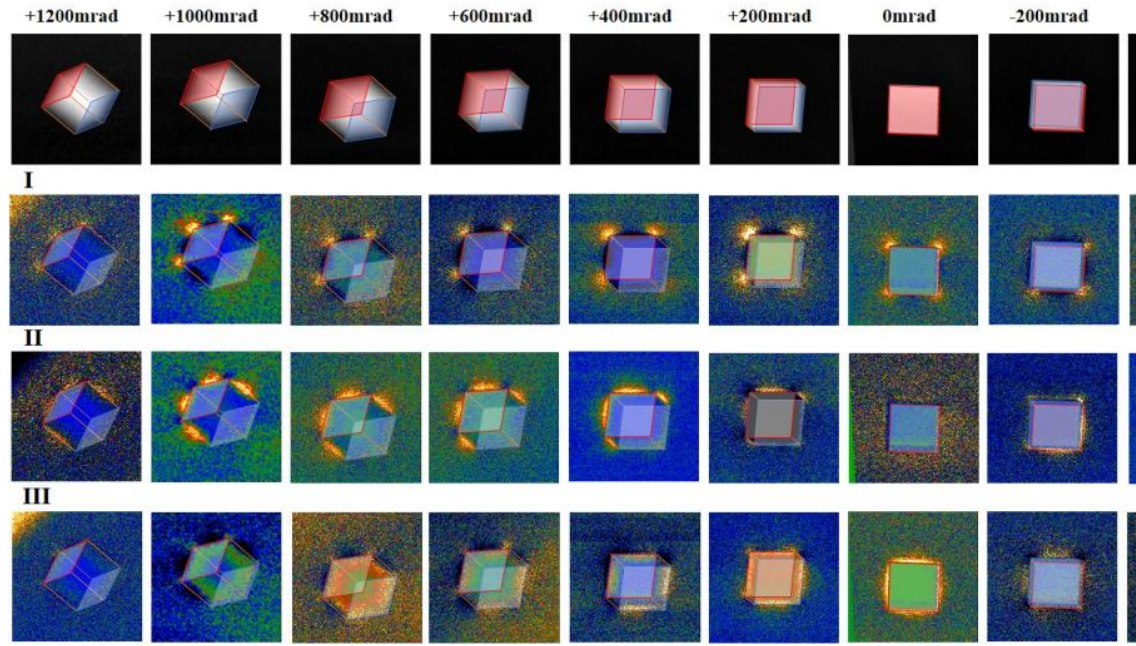

$-400 \mathrm{mrad}$

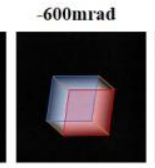

$-800 \mathrm{mrad}$

$-1000 \mathrm{mrad}$
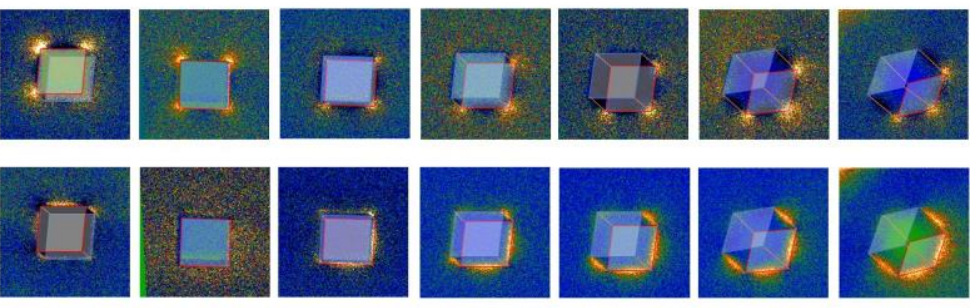

Figure 1. Figure.1 (a) Typical spectra for the three different $\mathrm{SPhP}$ modes on $\mathrm{MgO}$ cube surface. (b) 2D mapping at a series of tilt angles from $+1200 \mathrm{mrad}$ to $-1000 \mathrm{mrad}$. From top row to bottom, HAADF images at 12 different tilt angles, the corresponding filtered maps extracted at energies of the three main surface phonon modes. The color scale of each filtered map is adjusted independently to optimize the visualization of the signals.

\section{References}

1. K. Kliewer, R. Fuchs, Theory of dynamical properties of dielectric surfaces, vol. 27 (Wiley, UK, 1974).

2. J. Greffet, et al., Nature 416, 61 (2002).

3. Y. Wu, et al., Science Advances 6 (2020).

4. B. Song, et al., Nature Nanotechnology 10, 253 (2015).

5. O. L. Krivanek, et al., Nature 514, 209 (2014).

6. M. J. Lagos, A. Trügler, U. Hohenester, P. E. Batson, Nature 543, 529 (2017).

7. F. S. Hage, G. Radtke, D. M. Kepaptsoglou, M. Lazzeri, Q. M. Ramasse, Science 367, 1124 (2020).

8. Nicoletti et al., Nature 502, 7469 (2013).

9. Hörl et al., Nature Comm. 8, 1 (2017).

10. X. Li et al., accepted in Science (2021). 